\title{
Salubrinal alleviates traumatic spinal cord injury through suppression of the eIF2 $\alpha /$ ATF4 pathway in mouse model
}

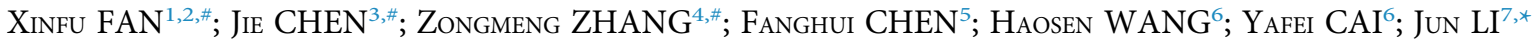 \\ ${ }^{1}$ Department of Orthopedics, Affiliated Hospital, Anhui College of Traditional Chinese Medicine, Wuhu, 241000, China \\ 2 Department of Orthopedics, Wuhu Hospital of Traditional Chinese Medicine, Wuhu, 241000, China \\ 3 The Second People's Hospital of Wuhu, Wuhu, 241000, China \\ 4 The School of Biomedical and Pharmaceutical Sciences, Guangdong University of Technology, Guangzhou, 510006, China \\ 5 College of Animal Science and Technology, Nanjing Agricultural University, Nanjing, 210095, China \\ 6 The Forth Hospital of Taizhou, Taizhou, 225300, China \\ 7 College of Life Sciences, Anhui Provincial Key Laboratory of the Conservation and Exploitation of Biological Resources, Anhui Normal University, Wuhu, 241000, China
}

Key words: SCI, Apoptosis, Endoplasmic reticulum stress, Salubrinal

\begin{abstract}
Spinal cord injury (SCI) remains an intractable clinical challenge of neurosurgery, it can be divided into two stages: uncontrollable primary injury induced by mechanical damage and controllable secondary injury regulated by continuous cell death. The apoptosis was the one of most important events in secondary injury, previous studies revealed that excessive endoplasmic reticulum (ER) stress breaks down the homeostasis and triggers apoptosis in the spinal cord. To deter or alleviate the secondary jury, we screen one of fat-soluble compounds, salubrinal, which was an inhibitor of eIF2 $\alpha$ dephosphorylation can repair SCI by inhibiting ER stress in mice after SCI. Administration of salubrinal effectively represses apoptosis, protects neuronal cell, and promotes the restoration of locomotor function in mice SCI models. Furthermore, the level of phosphorylated eIF2 $\alpha$ was raised in the presence of salubrinal, but the protein expression of ATF4 and CHOP was downregulated. Unexpectedly, transcriptional expression of CHOPregulated pro-apoptotic genes was decreased. These data suggest salubrinal suppress ER stress by targeting eIF2 $\alpha /$ ATF4 pathways and reduces cell death after SCI. It is suggested that the mitigation of secondary lesion by inhibiting ER stress induced apoptosis in the early phase of SCI is promising treatment strategy.
\end{abstract}

\section{Introduction}

Traumatic spinal cord injury (SCI) usually causes intense external intense stimulus, a severe type of high disability and incurable disease of the central nervous system (CNS). Allen first proposed the two-stage damage theory, which classified acute SCI into primary and secondary injuries (Allen, 1914; Allen, 1911). The primary injury changes the neurovascular microenvironment at the injury site, generating a secondary injury (Choo et al., 2008). Although studies have recognized secondary injury as the main cause for the loss of bodily function after SCI, the specific pathological mechanism remains unclear. Recent studies have shown that secondary injury is closely correlated with endoplasmic reticulum (ER) stress (Ohri et al., 2013; Ohri et al., 2011; Valenzuela et al., 2012).

\footnotetext{
*Address correspondence to: Jun Li, lijunplant@163.com

${ }^{\#}$ These authors have contributed equally to this work

Received: 12 July 2021; Accepted: 16 August 2021
}

Identifying the mechanism of ER stress and its role in the secondary injury of the spinal cord will further assist in the discovery of therapeutic targets, thus promoting the functional recovery of patients subjected to SCI.

ER stress is a series of adaptive regulatory responses under stress, which can determine cell survival of cells according to the strength and duration of stress, thus physical function. Under stress, the local microcirculatory disorder of the spinal cord leads to an insufficient supply of energy, and the accumulation of unfolded or misfolded proteins in the ER lumen activates the unfolded protein response (UPR) (Schroder and Kaufman, 2005a, 2005b). The molecular chaperone glucose-regulated protein-78 (Grp78), couples with phosphorylated protein kinase-like ER kinase (PERK), inositol-requiring enzyme 1 (IRE1) and activating transcription factor 6 (ATF6), respectively (Harding et al., 2000). Then, Grp78 combines unfoldable or misfolded proteins to induce degradation after the protein has left the original binding site. In ER stress, the PERK/eIF2 $\alpha$ 
pathway is involved in the UPR, where it inhibits protein synthesis by promoting phosphorylation of eukaryotic translation initiation factor $2 \alpha$ (eIF2 $\alpha)$. Subsequently, activation of activating transcription factor 4 (ATF4) enters the nucleus to bind the promoter ER stress response element components and induce the expression of ER-stressassociated genes (Ron and Walter, 2007). Therefore, these measures stabilize ER homeostasis to promote cell survival. As an extension of ER stress, they promote the apoptotic process by inducing the expression of C/EBP homologous protein $(\mathrm{CHOP})$ and increasing the transcriptional expression of proapoptotic genes (Marciniak et al., 2004).

Previous studies have reported promoting the homeostasis in ER can reduce the loss of oligodendrocyte, restoring the homeostasis of the ER and activating UPR improves the function recovery after SCI (Ohri et al., 2013; Ohri et al., 2011; Valenzuela et al., 2012). Similarly, some drugs like amiloride, valproicacid, and phenylbutyrate (PBA) have been successful in improving the function recovery in mouse models by inhibiting ER stress (Hosoi et al., 2010; Lee et al., 2014; Mizukami et al., 2010; Penas et al., 2011; Zhou et al., 2016). Our previous findings have demonstrated Zbtb38, a zinc finger protein family member, plays a negative regulatory role in the apoptosis mediated by SCI. In an in vitro experiment, Cai et al. (2012, 2017) argued that ATF4 reduced the amount of Zbtb38 promoters bound to inhibit the expression of Zbtb38, and that the decreased expression of Zbtb38 promoted ER-stress-mediated apoptosis of cells. Also, our previous studies have revealed treatment of nimodipine and tauroursodeoxycholic acid (TUDCA) exert a good therapeutic effect in SCI mouse models (Cai et al., 2011; Zhang et al., 2017). Therefore, drug intervention in ER stress to treat diseases may also be an effective treatment for SCI.

Salubrinal is a newly discovered compound that can inhibit eIF2 $\alpha$ dephosphorylation and maintain its phosphorylation state, thereby inhibiting protein translation and reducing protein synthesis, maintaining ER homeostasis, and adapting to intracellular stress to prevent the induction of ER stress in cells (Boyce et al., 2005). Salubrinal does not protect against apoptotic stimuli unrelated to ER stress and is a useful tool for studying ER stress. Salubrinal has been demonstrated to have effective therapeutic and cytoprotective effects in various disease models, and to perform well, performance especially in neuroprotection (Anuncibay-Soto et al., 2016; Barreda-Manso et al., 2017; Huang et al., 2012; Rubovitch et al., 2015), but little is known about its function in SCI. In this study, we established a mouse model of SCI and evaluated of salubrinal based on a series of studies after intraperitoneal administration of salubrinal. Our work reveals that salubrinal, as an ER stress inhibitor, effectively inhibits secondary SCI by targeting the reduction of ER stress and promotes the recovery of motor function in mice.

\section{Materials and Methods}

\section{Animal model of SCI and drug treatment}

All animal experimental procedures were approved by the Academic Ethics Committee of Anhui Normal University and complied with the ARRIVE guidelines and carried out in the National Institutes of Health guide for the care and use of laboratory animals. Eight weeks old Kun-Ming (KM) male mice weighing 30-35 g were provided by Qinglongshan animal breeding grounds. Mice were maintained in clean animal house with stationary temperature $\left(23-25^{\circ} \mathrm{C}\right)$ and $12 \mathrm{~h}$ light/ dark cycles, received free access to sufficient food and water.

The building procedure of SCI model was performed using compression method as described previously (Cai et al., 2011; Cai et al., 2012; Zhang et al., 2017). Mice were anaesthetized with $10 \%$ chloral hydrate ( $4 \mathrm{mg} / \mathrm{kg}$, i.p.). Then, the dorsal zone of mouse was incised along the midline; laminectomy was performed at the level of T7-T9 vertebras to expose the spinal cord. At this level, the spinal cord was compressed with a $30 \mathrm{~g}$ weight drop for $3 \mathrm{~min}$. The sham group mice were subjected the same surgical procedure but without compression injury. The incision sites were sutured, and mouse was alone maintained in cage. To prevent from urinary retention, we regularly assisted mice to urinate, until the mice regained the automatic micturition reflex.

Salubrinal was purchased from Solarbio (Beijing, China) and dissolved in DMSO (MP Biomedicals) at a concentration of $20 \mathrm{mg} / \mathrm{mL}$ and further diluted in corn oil. Mice were immediately administrated through intraperitoneal injection at a dose of $1 \mathrm{mg} / \mathrm{kg}$ body weight after surgery. The control mice were treated with equivalent vehicle alone.

\section{Behavioral assessment}

The Basso mouse scale (BMS) was used for the comprehensive rate of motor function after SCI as previously described (Basso et al., 2006). Hind limb function could be evaluated according to the BMS motor function rating gauge. The scale ranges from 0 (complete paralysis) to 9 (normal movement of the hind limbs). For the BMS score, the mice were placed in an open field $(50 \times 50 \times 30 \mathrm{~cm})$ divided into 25 sections $(10 \times$ $10 \mathrm{~cm}$ each). The mice were allowed to walk about in the open field for several days before SCI surgery to acclimatize themselves to the apparatus. The open field test was recorded throughout by video camera and was assessed by repeated observation. The mice were observed for $4 \mathrm{~min}$ by three independent observers who were unaware of the groupings and who all received the same score training. Scores were obtained for each hind limb and averaged for the three observers on each day. The strength of hind limb recovery was evaluated with inclined plane angles. The inclined plane test was placed on a rubber plate, and then gradually raised on one side of the plane, and the maximum angle at which mice could remain stable for at least $5 \mathrm{~s}$ was noted. Each mouse was assessed three times, and the average was taken. The mean of all mice in each group was the final result. We followed a previously mentioned procedure to analyze the data.

\section{Histology and immunochemistry}

Mice were anesthetized with chloral hydrate $(60 \mathrm{mg} / \mathrm{kg}$, i.p.) and transcardially perfused with $4 \%$ polyformaldehyde in $0.1 \mathrm{M}$ PBS. The lesion epicenter of the spinal cord was dissected/removed and fixed in $10 \%$ neutral buffered formalin solution for $24 \mathrm{~h}$. The fixed tissue was dehydrated in graded alcohol, transparentized in xylene and embedded in paraffin. 
The samples were transversely sectioned at $5 \mu \mathrm{m}$ thickness and the sections were mounted on microscope slides.

For immunohistochemistry analyses, the protocol was performed according to the manufacturer's instructions (Boster Biological Technology, Wuhan, China). Spinal cord sections were deparaffinized, rehydrated and performed antigen retrieval with microwave method. The sections blocked with $3 \% \mathrm{H}_{2} \mathrm{O}_{2}$ for $15 \mathrm{~min}$, incubated with protein block solution containing 5\% normal horse serum and $1 \%$ normal goat serum in PBS for $30 \mathrm{~min}$ at $37^{\circ} \mathrm{C}$. Then sections were incubated with primary antibody at 4 overnight, followed by horseradish peroxidase-conjugated secondary antibody incubation for $30 \mathrm{~min}$. Sections were incubated with developing solution (diaminobenzidine, $\mathrm{DAB})$ and counter stained with hematoxylin (Boster).

\section{TUNEL staining}

Apoptosis was assayed with in situ Cell Death Detection Kit (Roche, Indianapolis, USA) according to instructions. For tissues, paraffin sections were deparaffinized according to standard procedures. Antigen retrieval was performed in citrate buffer with $750 \mathrm{~W}$ microwave irradiation for $20 \mathrm{~min}$, and sections were blocked with Tris- $\mathrm{HCl}(0.1 \mathrm{M}, \mathrm{PH} 7.5)$ containing $3 \%$ BSA and $20 \%$ normal bovine serum for $30 \mathrm{~min}$ at $37^{\circ} \mathrm{C}$. After washing with $\mathrm{PBS}$, sections were incubated with $50 \mu \mathrm{L}$ TUNEL reaction mixture for $1 \mathrm{~h}$ at $37^{\circ} \mathrm{C}$ in a humidified atmosphere in the dark. Finally, the slides were mounted with the DAPI mounting medium (Solarbio, Beijing, China). Slides were observed captured with the fluorescence microscope (Olympus, BX61), and positive cells were counted from digitally captured images.

\section{Immunofluorescence staining}

Spinal cord sections were deparaffinized and rehydrated. Sections were carried out antigen retrieval in sodium citrate containing $0.05 \%$ Tween 20 with microwave method, and permeabilization in PBS containing $0.2 \%$ Triton X-100. Then sections were blocked with PBS containing $10 \%$ goat serum and $1 \%$ BSA, incubated with primary antibody in blocking buffer overnight at $4^{\circ} \mathrm{C}$, followed secondary antibody conjugated $\mathrm{Cy} 3$ incubation for $2 \mathrm{~h}$ at room temperature. After washing with PBS, sections were mounted with antifade mounting medium with DAPI. The images were obtained using confocal microscope.

$R N A$ extraction and quantitative RT-PCR ( $q R T-P C R$ )

Total RNA was extracted from injured spinal cord tissues by using Trizol reagent (Invitrogen, Carlsbad, USA); isolated RNA was reversely transcribed based on the FastQuant RT Kit (Tiangen, Beijing, China). Amplification and real-time detection were performed on a CFX96 instrument (Bio-Rad, Hercules, USA) by using the Super Real PreMix Plus (Tiangen). The improved four steps reaction was used: $95^{\circ} \mathrm{C}$ $15 \mathrm{~min} ; 95^{\circ} \mathrm{C} 10 \mathrm{~s}, 60^{\circ} \mathrm{C} 32 \mathrm{~s}, 72^{\circ} \mathrm{C} 32 \mathrm{~s}, 85^{\circ} \mathrm{C} 6 \mathrm{~s}$, for 40 cycles; the melting curve analysis ranging from $60^{\circ} \mathrm{C}$ to $95^{\circ}$ $\mathrm{C}$, gradually increasing at a speed of $0.5^{\circ} \mathrm{C}$ every $10 \mathrm{~s}$. Primers used for real-time RT-PCR are listed in Table 1. The relative expression levels of each targeted gene were normalized by subtracting the corresponding mouse $\beta$-actin threshold cycle (CT) values by using the $\Delta \Delta \mathrm{CT}$ comparative method.

\section{Protein extraction and western blot analysis}

For protein extraction, the injured segments of spinal cord $(5 \mathrm{~mm})$ were collected and homogenized in protein lysis solution (1\% NP-40, 1\% Sodium deoxycholate, 0.1\% SDS) containing a protease inhibitor PMSF, incubated for $1 \mathrm{~h}$ at $4^{\circ} \mathrm{C}$. The protein concentration was determined using bicinchonininc acid (BCA) protein assay kit (Thermo Fisher Scientific, Waltham, MA, USA). The proteins were diluted to $1 \mu \mathrm{g} / \mu \mathrm{L}$ with protein loading buffer and denatured in metal bath. Then the equivalent proteins were separated by SDSPAGE and transferred on Immobilon-p Transfer Membrane (Merck Millipore, Darmstadt, Germany) with the wet electrical transfer method. The membranes were blocked with $5 \%$ BSA in TBS containing $0.1 \%$ Tween-20 for $1 \mathrm{~h}$ at room temperature; followed by the primary antibody incubation overnight at $4^{\circ} \mathrm{C}$ and the secondary antibody for $1 \mathrm{~h}$ at room temperature. The bands were developed/detected with ECL detection system according to the manufacturer's protocol (TransGen Biotech, Beijing, China) and chemiluminescence signal was visualized by exposure to Kodak X-Omat AR film (Rochester, NY). The gray intensities of bands were measured

TABLE 1

qPCR primer sequence

\begin{tabular}{lll}
\hline Gene & Forward primer (5'-3') & Reverse primer (5’-3') \\
\hline GAPDH & AACTTTGGCATTGTGGAAGG & ACACATTGGGGGTAGGAACA \\
Grp78 & ACTTGGGGACCACCTATTCCT & ATCGCCAATCAGACGCTCC \\
CHOP & GCATGAAGGAGAAGGAGCAG & ATGGTGCTGGGTACACTTCC \\
ATF4 & AGACACCGGCAAGGAGGAT & CATCCAACGTGGTCAAGAGC \\
Bak & ACTGTGTTAACTCCTGCCCG & GCAGCAAGCTACTCAGACGA \\
Bax & AAAATGGCATCTGGACAAGG & AAGATGCTGTTGGGTTCCAG \\
Bim & TGCAGAGGATGATTGCTGAC & GATCAGCTCGGGCACTTTAG \\
DR5 & GCGAACTCTGTGCATTCGTC & ACCGGAACCAGCAACTTCTT \\
Puma & GCCCAGCAGCACTTAGAGTC & TGTCGATGCTGCTCTTCTTG \\
Noxa & GGCAGAGCTACCACCTGAGT & TTGAGCACACTCGTCCTTCA \\
\hline
\end{tabular}


using ImageJ software (Bethesda, MD, USA) and were normalized for GAPDH or $\beta$-tubulin. Anti-GAPDH (TA-08; $1: 1000)$, anti- $\beta$-tubulin (TA-08; $1: 1000)$, and the secondary antibody (anti-mouse-HRP ZB-5305 and anti-rabbit-HRP ZB-5301; 1:5000) were purchased from ZSGB-Bio. eIF2 $\alpha$, P-eIF2aand Grp78 (3177; 1:1000) were from Cell Signaling Technology. ATF4 (BM5179; 1:100) and CHOP (BM4962; 1:100) was obtained from Boster Biological Technology.

\section{Statistical analyses}

Statistical analysis was conducted using ANOVA and Student's $t$-test, using GraphPad Prism software. The data shown are representative of at least three independent experiments. A value of $P<0.05$ was considered to indicate a statistically significant difference.

\section{Results}

Administration of salubrinal promote the recovery of motor function after SCI

To monitor the effect of salubrinal on the motor recovery, we performed the BMS and inclined plane test scores from 1 to 14 days post SCI surgery. We detected the spinal cord injured mice suffer paralysis of the lower body and the incapacitated lower limbs, but the motor function was gradually recovered in the following days. The results of BMS shown no significant difference in recovery of motor function at 1 and 3 days after administration of salubrinal compared with the vehicle group (Fig. 1A). The average BMS score for salubrinal-treated group increased to 6.9 on 7th day. In contrast, vehicle-treated group show the average BMS score of 5.3. Similarly, the BMS subscore show the same changing tendency (Fig. 1A). The average angle of inclination for vehicle- and salubrinal-treated group was $53^{\circ}$ and $60^{\circ}$ in inclined plane test on 10th day (Fig. 1B). Meanwhile, the strength of the lower limbs was restored, and the mice exercised frequently in the salubrinal group. Therefore, the results of behavior examination show that salubrinal promotes the recovery of motor function in mice after SCI.

Salubrinal reduce apoptosis and promote the survival of neurons in the injury location

The cascade of secondary damage leads to apoptosis, and then impairs nerve conduction and reflex function in the injured area. Thus, we next examined whether salubrinal can protect spinal cord cells at lesion location. After obtaining transected tissue sections at the lesion center, TUNEL was used to detect apoptosis. Primarily, apoptotic cells mainly existed on the white matter region at the central part of the lesion after SCI (Fig. 2A). A growing number of dead cells were observed in gray matter on the 7 th day. But compared with the vehicle treated group, the administration of salubrinal significantly decreased the number of apoptotic cells on the 3rd and 7th day (Fig. 2B). Hence, our works revealed salubrinal inhibits the proceeding of apoptosis after SCI.

To demonstrate the protection effective of salubrinal for neuronal cells, we further used NeuN immunofluorescence to label the posterior neurons of the spinal cord. After salubrinal administration, neuronal cells were protected in compare with vehicle group (Fig. 3A). Salubrinal-treated mice showed significantly more the number of survival neuron compared to vehicle-treated mice on the 3rd and 7th day (Fig. 3B). Therefore, we propose that salubrinal has a neuroprotective effect on SCI and effectively inhibits neuronal death.

\section{Salubrinal did not affect the proliferation of astrocytes}

A large number of astrocytes are accumulated at the site of injury after SCI. We implemented GFAP immunohistochemistry to label the astrocytes in damage zone of the posterior gray. Initially, the proliferation of astrocytes was observed after SCI. Then a growing body of astrocytes are produced in the injured area on the 7th day, and the cell bodies become larger (Fig. 4A). Interestingly, salubrinal did not significantly alter the astrocyte population compared with the vehicle-treated group at each time point (Fig. 4B).

\section{Salubrinal suppresses ER stress induced apoptosis by targeting eIF2 $\alpha$-ATF4 signal}

To demonstrate salubrinal is a specific ER stress inhibitor that acts on eIF2 $\alpha$ in the PERK-eIF2 $\alpha$-ATF4 pathway, we further analyzed the levels of gene associated ER stress. Notably, the phosphorylation protein of eIF2 $\alpha$ was significantly enhanced after salubrinal administration, but there was not distinctly change in the total eIF2 $\alpha$ protein (Fig. 5A). Although the expression level of Grp78 was higher on the first day, it was followed protein expression was lower on the $3 \mathrm{rd}$ and 7 th day compared with the vehicle-treated group (Fig. 5B). The
A

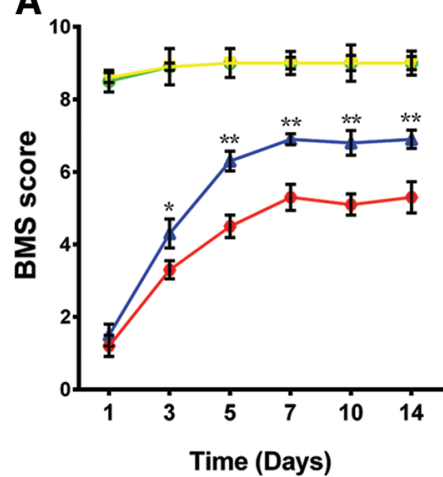

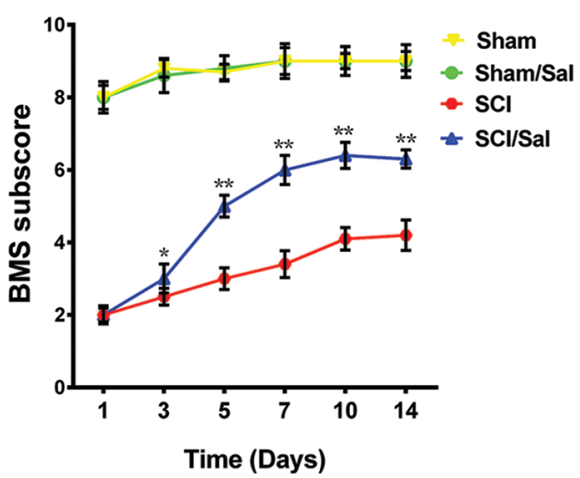

B

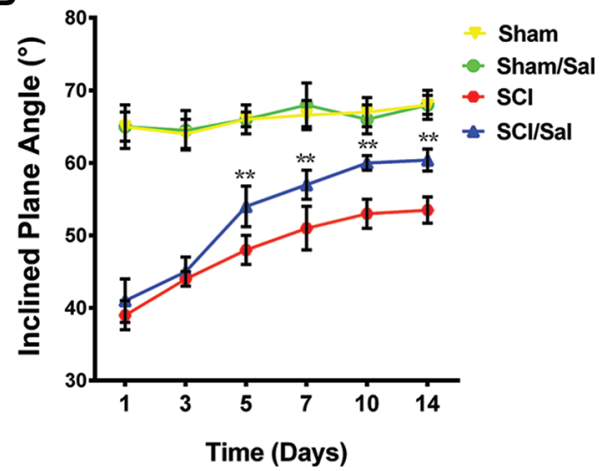

FIGURE 1. Administration of salubrinal promote the recovery of motor function after SCI. The behavioral assessment was determined from 1 to 14 days after injury. (A) is BMS score; (B) is the inclined plane test scores. Data are represented as means \pm SEM, sham control $(\mathrm{N}=10)$, vehicle treated $(\mathrm{N}=7)$ and salubrinal treated $(\mathrm{N}=8),{ }^{\star} P<0.05,{ }^{* *} P<0.01$. 
A
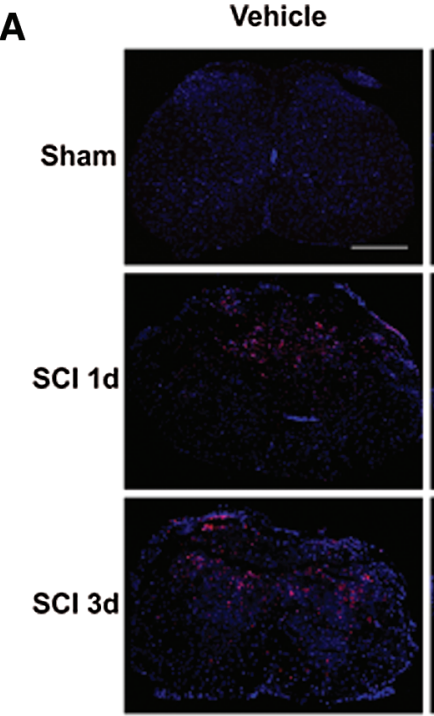

SCI 7d

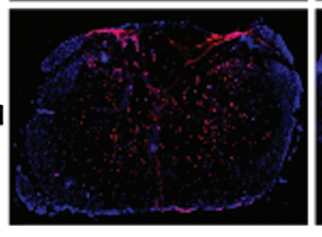

A

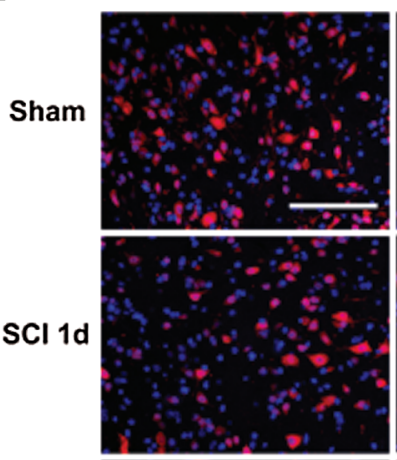

Vehicle

SCI 3d

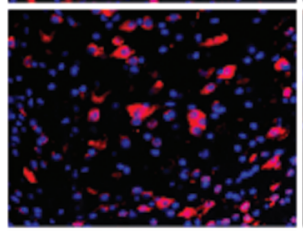

SCI 7d

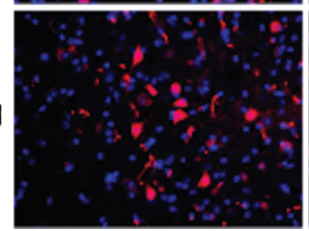

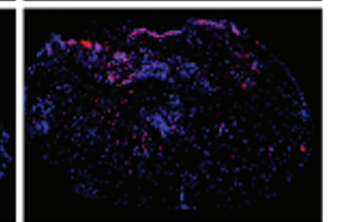

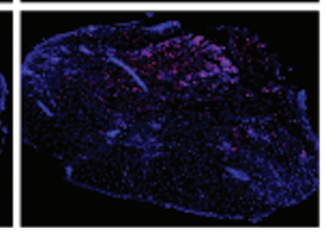

\section{Salubrinal}
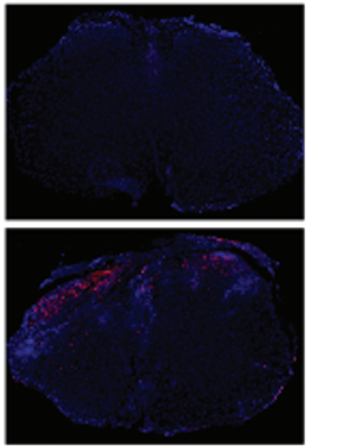

Salubrinal
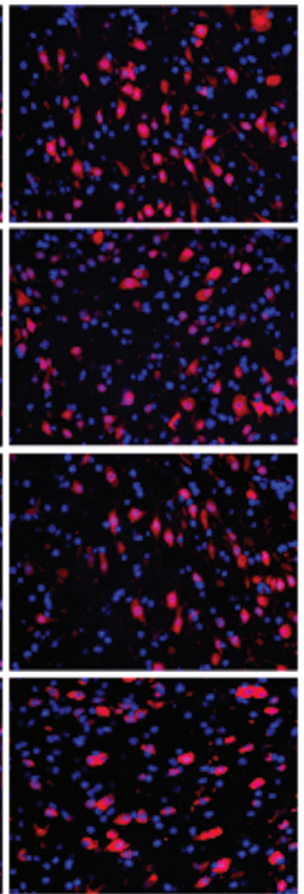

B

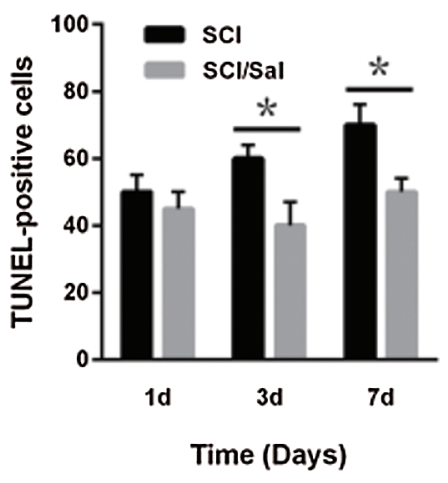

FIGURE 2. Salubrinal reduce apoptosis after spinal cord injury. (A) The TUNEL staining in sham control, vehicle treated and salubrinal treated mice respectively at $1,3,7$ days post SCI. Apoptotic cells are marked as red, the nuclei are labeled with DAPI (blue). Scale bar, $1 \mathrm{~mm}$. (B) The quantitative analysis of TUNELpositive cells in three groups. Data are represented as means $\pm \mathrm{SEM}, \mathrm{N}=3$ per each group, ${ }^{\star} P<0.05$.
B

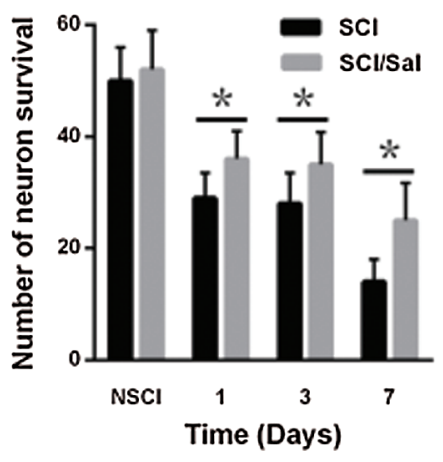

FIGURE 3. Salubrinal promote the survival of peripheral neurons in the injured area in mice. (A) Representative immunofluorecent images of $\mathrm{NeuN}$ at the epicenter of lesion in sham control, vehicle treated and salubrinal treated mice respectively post SCI at $1,3,7$ days. The neurons are marked as red, the nuclei are labeled with DAPI (blue). Scale bar, $50 \mu \mathrm{m}$. (B) The quantitative analysis of the survival neurons in three groups. Data are represented as means \pm SEM, $\mathrm{N}=3$ per each group, ${ }^{\star} P<0.05$.

protein level of ATF4 and CHOP were significantly decreased, reduced dramatically on the 3rd day especially (Fig. 5). Next, the expression of ATF4-CHOP pathway-related genes was detected by qRT-PCR. Corresponding with the protein level, the transcriptional expression of Grp78, ERdj4, ATF4 and CHOP was decreased after salubrinal treatment (Fig. 6). This suggests that salubrinal acts on ER stress by targeting eIF2 $\alpha$-ATF4 signal.

We next examined the expression of Bcl-2 pro-apoptotic gene family including Bax, Bak, Bim, Puma, and Noxa by
qRT-PCR analysis. It was found that the transcription expression of pro-apoptotic gene was down-regulated after salubrinal administration compared with the vehicle-treated group (Fig. 7). It has been shown that ATF4 and CHOP are transcriptional regulatory factors of the pro-apoptotic genes of the Bcl-2 family. In addition, we observed that salubrinal attenuate the protein levels of ATF4 and CHOP (Fig. 5). These results suggest that salubrinal reduces the expression of the pro-apoptotic gene by downregulating the protein expression of ATF4 and CHOP. 
A

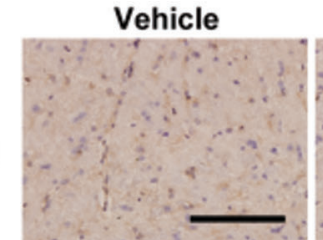

\section{Salubrinal}

Sham

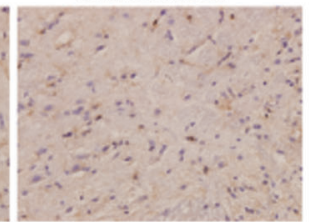

$\mathrm{SCl} 1 \mathrm{~d}$

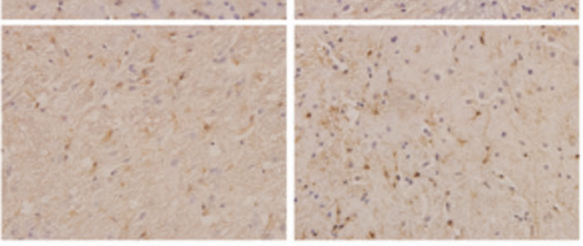

$\mathrm{SCl} 3 \mathrm{~d}$
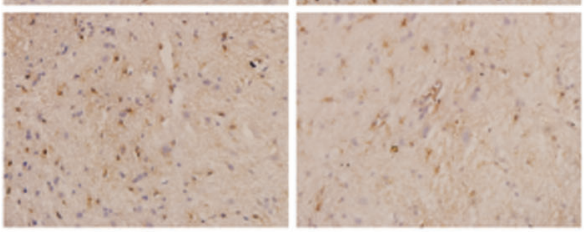

SCI 7d

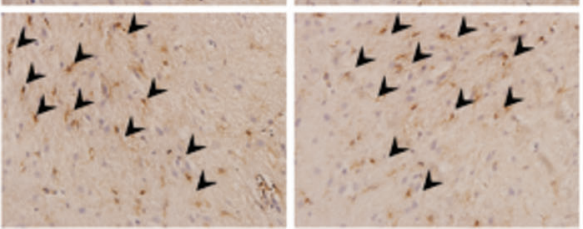

B

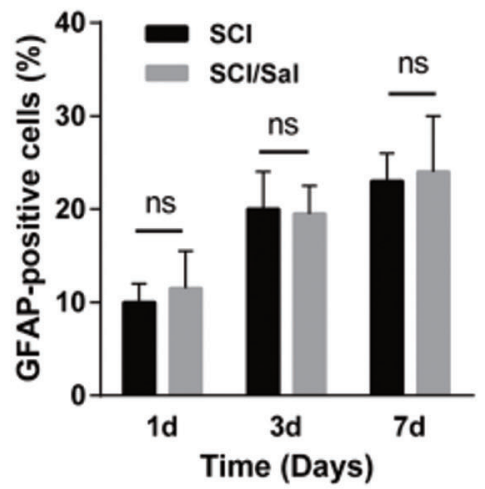

FIGURE 4. Salubrinal did not affect the proliferation of astrocytes. (A) Representative immunohistochemistry images of GFAP at the epicenter of lesion in sham control, vehicle treated and salubrinal treated mice respectively post SCI at 1, 3, 7 days. The black arrow indicates astrocytes. Scale bar, $50 \mu \mathrm{m}$. (B) The quantitative analysis of GFAP positive cells in three groups. Data are represented as means \pm SEM, $\mathrm{N}=3$ per each group.

A
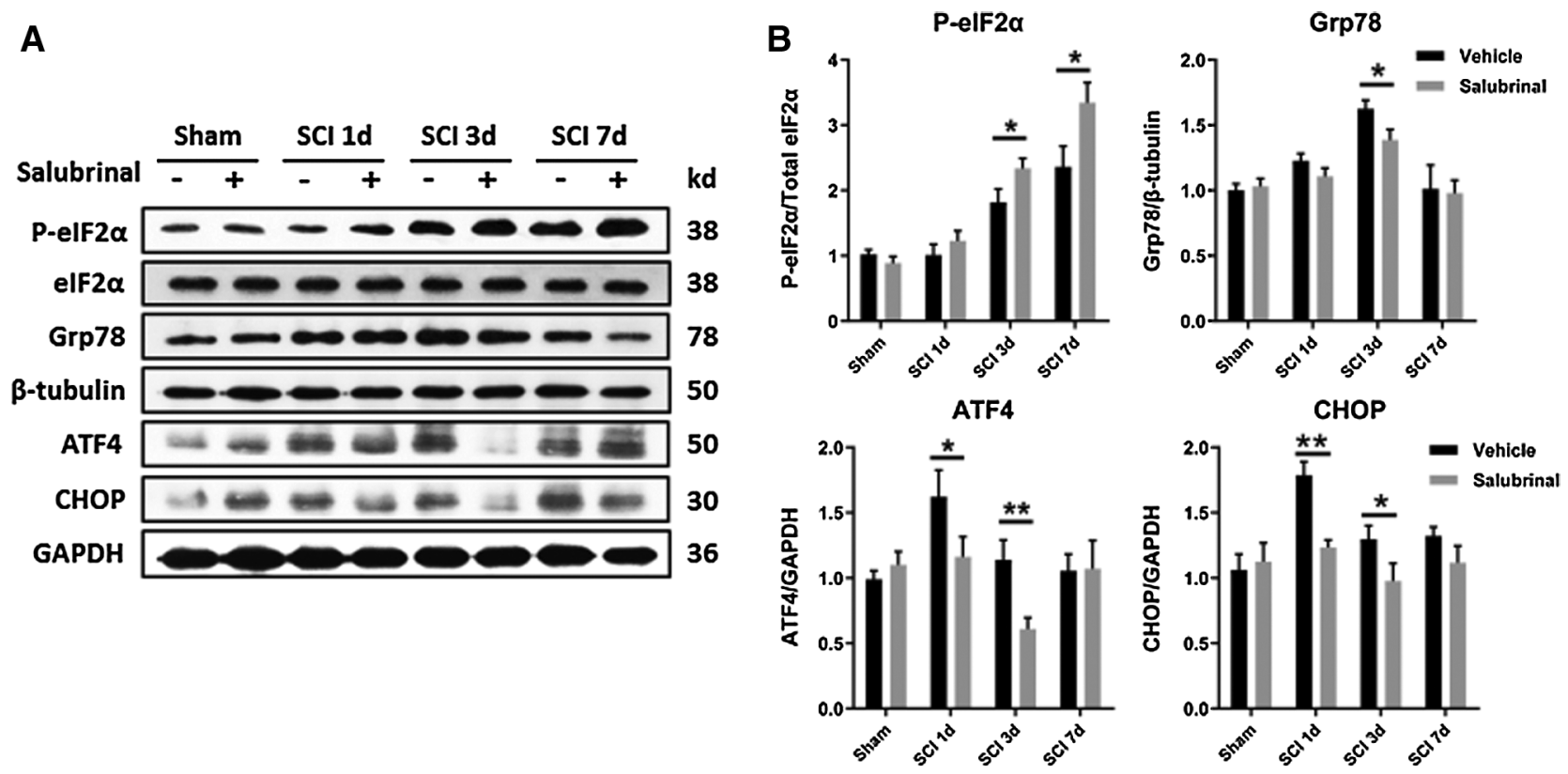

FIGURE 5. Salubrinal suppresses ER stress by targeting eIF2 $\alpha$-ATF4 signal. (A) Immunoblot analysis for eIF2 $\alpha$, p-eIF2 $\alpha$, Grp78, ATF4 and CHOP levels in sham control, vehicle treated and salubrinal treated mice respectively post SCI at 1, 3, 7 days. (B) The quantitative analysis of band densities was normalized to $\beta$-tubulin or GAPDH. Data are represented as means \pm SEM, $N=3$ per each group, ${ }^{\star} P<0.05,{ }^{\star *} P<0.01$.

\section{Discussion}

In clinical practice, the release of primary pathogenic factors and the emphasis on reducing or even avoiding secondary damage to neuronal cells are crucial factors in the treatment of SCI (Thuret et al., 2006). The aggravation of secondary damage gives rise to poorer functional recovery after SCI, and the death of a large number of spinal nerve cells leads to the emergence of irreversible damage (Choo et al., 2008). Therefore, inhibiting apoptosis caused by secondary injury is the first step in the treatment of SCI. In this paper, our work reveals that salubrinal modulates ER stress by targeting eIF2a-ATF4 signaling to reduce apoptosis, protect neurons and improve the recovery of motor function in a mouse model of SCI.

Notably, ER stress is triggered by the accumulation of unfolded or misfolded proteins in the ER and is a disorder of intracellular homeostasis (Schroder and Kaufman, 2005a). Cells activate the UPR in response to ER stress to protect themselves from harm. In recent years, army of studies has reported that ER stress is closely correlated with the occurrence and development of various diseases 

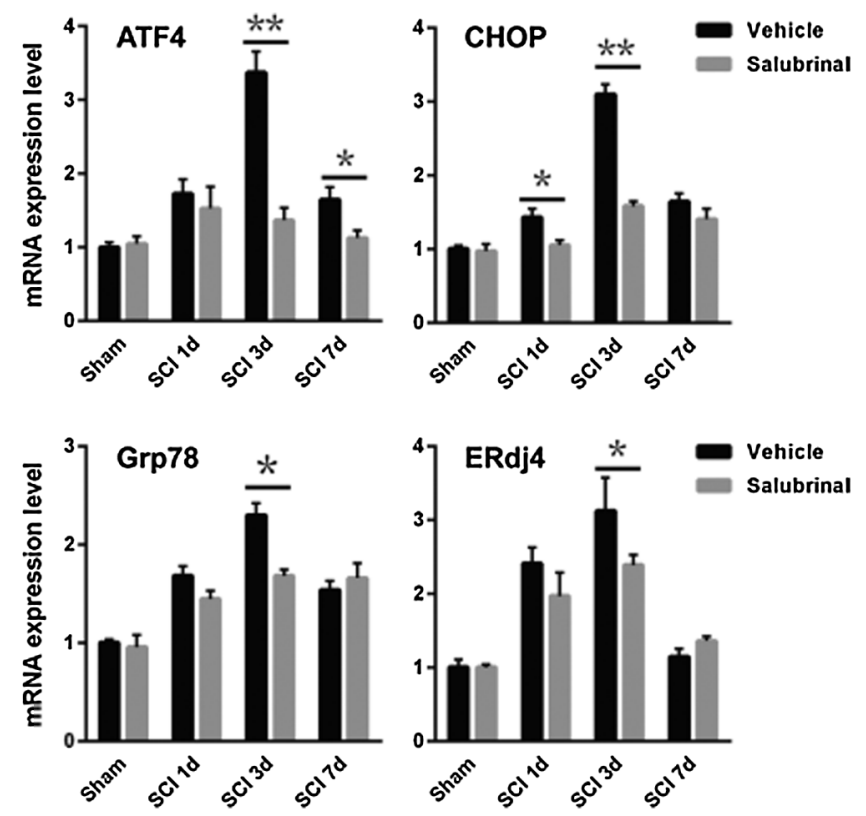

FIGURE 6. Salubrinal repress the transcriptional expression of gene associated with eIF2 $\alpha$-ATF4 pathway. The qRT-PCR analysis mRNA expression levels of the eIF2 $\alpha$-ATF4 signal gene ATF4, CHOP, Grp78 and ERdj4 in sham control, vehicle treated and salubrinal treated mice respectively post SCI at 1, 3, 7 days. Data are represented as means \pm SEM, $\mathrm{N}=3$ per each group, ${ }^{\star} P<0.05,{ }^{\star \star} P<0.01$.

(Aridor and Balch, 1999; Li et al., 2013; Minamino and Kitakaze, 2010; Valenzuela et al., 2016). Several relevant studies have provided evidence favoring the activation of ER stress in different animal SCI models, including the splicing of XBP1 mRNA, the induction and translocation of CHOP, and the up-regulation of Grp78 mRNA and protein (Ohri et al., 2011; Penas et al., 2007; Valenzuela et al., 2012). Furthermore, a recent study proposed that different types of cells produce discrepant sensitivity to ER stress responses after SCI (Penas et al., 2007). Our results suggest that the expression of ATF4, CHOP, and Grp78 serving as the ER stress markers were up-regulated, and the level of P-eIF2a was also elevated after SCI. Together with the current study, the signals associated with ER homeostasis were activated in lesions of the spinal cord in a mouse model.

Following SCI, as a pharmacological target, the homeostasis components of ER stress will be an effective therapeutic strategy for functional repair. Moreover, several studies have shown that small-molecule compounds that restore ER homeostasis may have therapeutic potential for ER stress-related CNS diseases (Valenzuela et al., 2016; Zhang et al., 2015). Several drugs that can improve the recovery of spinal cord motor function have been demonstrated to reduce ER stress levels. For example, amiloride is a potassium-sparing diuretic that significantly reduces ER stress-induced apoptosis and the expression of the pro-apoptotic transcription factor $\mathrm{CHOP}$, as well as increasing Grp78 levels and NG2-positive oligodendrocytes in the injured spinal cord (Hosoi et al., 2010; Kuroiwa et al., 2014). In addition, valproic acid (VPA), exogenous basic fibroblast growth factor (FGF) and nerve growth factor (NGF) also have an inhibitory effect on ER stress-induced apoptosis and improve motor recovery after SCI (Penas et al., 2011; Zhang et al., 2013). The results of this study demonstrate that salubrinal protects cells against apoptosis, improves the recovery of motor function, and protects neuronal cells. previous work demonstrated the neuroprotective effects of salubrinal on traumatic brain injury in a mouse model (Rubovitch et al., 2015). Interestingly, we observed that the proliferation of astrocytes was affected after SCI. Similarly, astrocytes were not activated in ATF4-deficient mice after SCI (Valenzuela et al., 2012). In fact, prominent studies have suggested that candidate factors in astrocytes protect them against ER stress. Therefore, astrocytes are less sensitive to ER stress in mice following SCI.

Promoting the balance of homeostasis in the ER can reduce oligodendrocyte loss, decrease cell death, and improve functional recovery after SCI (Ohri et al., 2013). As the downstream arm of the PREK-eIF2 $\alpha$ pathway, ATF4 and $\mathrm{CHOP}$ play an important role in regulating ER stress. ATF4
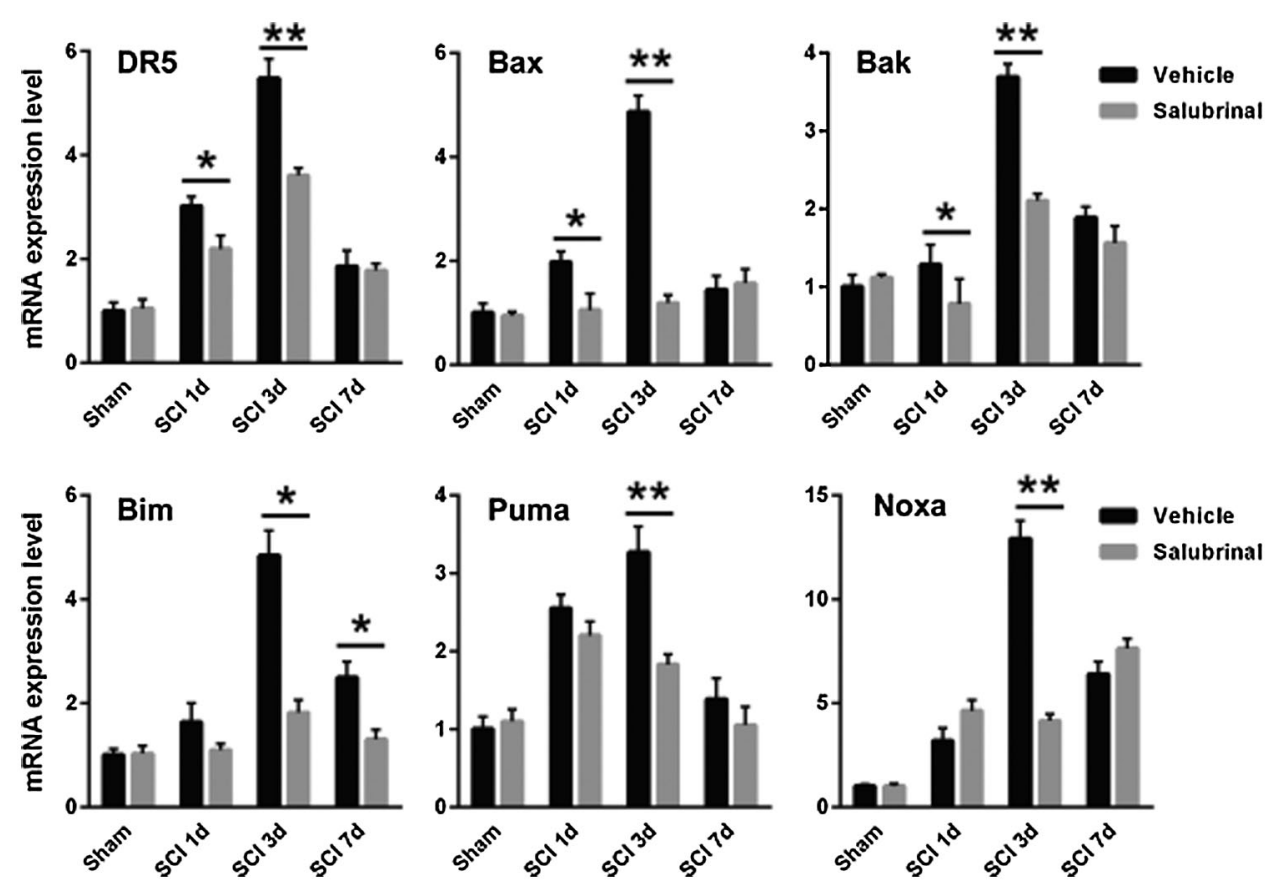

FIGURE 7. Salubrinal down-regulates the mRNA expression of pro-apoptotic gene. The qRT-PCR analysis mRNA expression levels of the pro-apoptotic gene Bak, Bax, Bim, Noxa, Puma, and DR5 in sham control, vehicle treated and salubrinal treated mice respectively post SCI at 1, 3, 7 days. Data are represented as means \pm SEM, N $=3$ per each group, ${ }^{\star} P<0.05,{ }^{\star *} P<0.01$. 
null mouse exhibited significantly the mitigation of motor recovery, and diminish the number of oligodendrocytes, but significant differences were not observed in the total number of neurons in compared with control mice in the lesion area after spinal cord hemisection (Valenzuela et al., 2012). Similarly, the motor recovery, the content of oligodendrocytes and white-matter sparing were enhanced in deletion of CHOP mice after spinal cord contusion (Ohri et al., 2011). Interestingly, when CHOP-deficient mice were sustained highintensity contusions, ER stress levels were evaluated in comparison with those exposed low-intensity contusions, but motor recovery was not ameliorated (Ohri et al., 2012). It is noteworthy that the reduction of ATF4 and CHOP served as therapeutic targets attenuates the pro-apoptotic pathway of ER stress and promotes the recovery of motor functions after SCI.

Salubrinal is a potent and specific ER stress inhibitor (Boyce et al., 2005). Related studies reported salubrinal reconstitute the balance between the ER homeostasis and the pro-apoptotic ER stress response branches (Ohri et al., 2013). After salubrinal treatment, the level of phosphorylated eIF2 $\alpha$ is elevated, which inhibits protein translation and reduces protein synthesis, alleviate the ER burden. Salubrinal also reduced the expression of ATF4 and CHOP. ER stress activates the CHOP protein, which can impair the antiapoptotic function of $\mathrm{Bcl}-2$ and regulate the transcriptional expression of the pro-apoptotic gene family of Bcl-2. In the stress state, these activated pro-apoptotic proteins promote apoptosis by leading to the destruction of ER membrane integrity and $\mathrm{Ca}^{2+}$ outflow (Oakes et al., 2005). Our work shown the pro-apoptotic genes of Bcl-2 family such as Bax, Bak, Noxa, and Puma, their transcriptional expression levels have been down-regulated after administration of salubrinal. Therefore, our findings reveal that its cytoprotection is associated with the restoration of ER homeostasis by heightening PERK-eIF2 $\alpha$ signaling and reducing expression of the pro-apoptotic genes correlated with ER stress (Fig. 8).

In conclusion, salubrinal, as an inhibitor of ER stress, effectively inhibits secondary spinal cord injury in mice by targeting the attenuation of ER stress and promotes the recovery of motor function in mice. Therefore, the

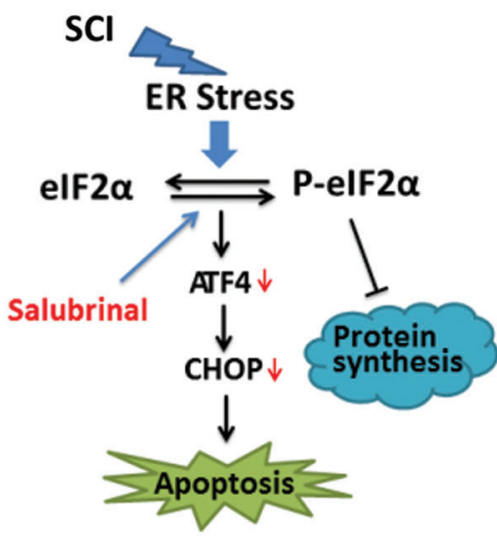

FIGURE 8. Model of the repair role of salubrinal in mouse of SCI. Salubrinal promote restoration of ER homeostasis by targeting eIF2 $\alpha$ ATF4 signal. Firstly, phosphorylating eIF2areduce the expression of proteins, reducing the ER burden. Secondly, downregulating the expression of Bax, Bak, Bim, Puma, Noxa, and other pro-apoptotic genes by reducing the protein expression of ATF4 and $\mathrm{CHOP}$. pharmacological regulation of ER stress by targeting PERKeIF2 $\alpha$ signal seems to be a new therapeutic approach. Our work is consistent with several studies confirmed pharmacological inhibition of ER stress can effectively treat SCI. In future, the research of drug intervention of ER stress plays beneficial role in treatment of SCI.

Availability of Data and Materials: All data generated or analyzed during this study are included in this published article or/are available from the corresponding author on reasonable request.

Ethics Approval: All animal experiments complied with the ARRIVE guidelines and were carried out according to the National Institutes of Health guide for the care and use of laboratory animals. All the study protocols were authorized by the Anhui Normal University Academic Ethics Committee (Approval Code: No. 180126014; Approval Date: January 26, 2018).

Author Contribution: The authors confirm contribution to the paper as follows: study conception and design: X. Fan, J. Li; data collection: F. Chen, H. Wang; analysis and interpretation of results: X. Fan, J. Chen, Z. Zhang; draft manuscript preparation: J. Chen, Y. Cai. All authors reviewed the results and approved the final version of the manuscript.

Funding Statement: This study was supported by Anhui Natural Science Foundation (1908085MC83), the National Natural Science Foundation of China (31970413), Wuhu Science and Technology Bureau Project (2019cg19).

Conflicts of Interest: The authors declare that they have no conflicts of interest to report regarding the present study.

\section{References}

Allen A (1914). Remarks on the histopathological changes in the spinal cord due to impact. An experimental study. Journal of Nervous and Mental Disease 41: 141-147. DOI 10.1097/ 00005053-191403000-00002.

Allen AR (1911). Surgery of experimental lesion of spinal cord equivalent to crush injury of fractured is location of spinal column. Journal of the American Medical Association 57: 878-880.

Anuncibay-Soto B, Perez-Rodriguez D, Santos-Galdiano M, Font E, Regueiro-Purrinos M et al. (2016). Post-ischemic salubrinal treatment results in a neuroprotective role in global cerebral ischemia. Journal of Neurochemistry 138: 295-306. DOI 10.1111/jnc.13651.

Aridor M, Balch WE (1999). Integration of endoplasmic reticulum signaling in health and disease. Nature Medicine 5: 745751. DOI 10.1038/10466.

Barreda-Manso MA, Yanguas-Casas N, Nieto-Sampedro M, RomeroRamirez L (2017). Neuroprotection and blood-brain barrier restoration by salubrinal after a cortical stab injury. Journal of Cellular Physiology 232: 1501-1510. DOI 10.1002/jcp.25655.

Basso DM, Fisher LC, Anderson AJ, Jakeman LB, Mctigue DM et al. (2006). Basso Mouse Scale for locomotion detects differences in recovery after spinal cord injury in five common mouse strains. Journal of Neurotrauma 23: 635-659. DOI 10.1089/ neu.2006.23.635.

Boyce M, Bryant KF, Jousse C, Long K, Harding HP et al. (2005). A selective inhibitor of eIF2alpha dephosphorylation protects 
cells from ER stress. Science 307: 935-939. DOI 10.1126/ science.1101902.

Cai Y, Fan R, Hua T, Liu H, Li J (2011). Nimodipine alleviates apoptosis-mediated impairments through the mitochondrial pathway after spinal cord injury. Current Zoology 57: 340349. DOI 10.1093/czoolo/57.3.340.

Cai Y, Li J, Yang S, Li P, Zhang X et al. (2012). CIBZ, a novel BTB domain-containing protein, is involved in mouse spinal cord injury via mitochondrial pathway independent of p53 gene. PLoS One 7: e33156. DOI 10.1371/journal.pone.0033156.

Cai Y, Li J, Zhang Z, Chen J, Zhu Y et al. (2017). Zbtb38 is a novel target for spinal cord injury. Oncotarget 8: 45356-45366. DOI 10.18632/oncotarget.17487.

Choo AM, Liu J, Dvorak M, Tetzlaff W, Oxland TR (2008). Secondary pathology following contusion, dislocation, and distraction spinal cord injuries. Experimental Neurology 212: 490-506. DOI 10.1016/j.expneurol.2008.04.038.

Harding HP, Novoa I, Zhang Y, Zeng H, Wek R et al. (2000). Regulated translation initiation controls stress-induced gene expression in mammalian cells. Molecular Cell 6: 10991108. DOI 10.1016/S1097-2765(00)00108-8.

Hosoi T, Kume A, Otani K, Oba T, Ozawa K (2010). A unique modulator of endoplasmic reticulum stress-signalling pathways: The novel pharmacological properties of amiloride in glial cells. British Journal of Pharmacology 159: 428-437. DOI 10.1111/j.1476-5381.2009.00544.x.

Huang X, Chen Y, Zhang H, Ma Q, Zhang YW et al. (2012). Salubrinal attenuates $\beta$-amyloid-induced neuronal death and microglial activation by inhibition of the NF- $\mathrm{KB}$ pathway. Neurobiology of Aging 33: e1009-e1017.

Kuroiwa M, Watanabe M, Katoh H, Suyama K, Matsuyama D et al. (2014). Effect of amiloride on endoplasmic reticulum stress response in the injured spinal cord of rats. European Journal of Neuroscience 40: 3120-3127. DOI 10.1111/ejn.12647.

Lee JY, Maeng S, Kang SR, Choi HY, Oh TH et al. (2014). Valproic acid protects motor neuron death by inhibiting oxidative stress and endoplasmic reticulum stress-mediated cytochrome $\mathrm{C}$ release after spinal cord injury. Journal of Neurotrauma 31: 582-594. DOI 10.1089/neu.2013.3146.

Li S, Yang L, Selzer ME, Hu Y (2013). Neuronal endoplasmic reticulum stress in axon injury and neurodegeneration. Annals of Neurology 74: 768-777. DOI 10.1002/ana.24005.

Marciniak SJ, Yun CY, Oyadomari S, Novoa I, Zhang Y et al. (2004). $\mathrm{CHOP}$ induces death by promoting protein synthesis and oxidation in the stressed endoplasmic reticulum. Genes \& Development 18: 3066-3077. DOI 10.1101/gad.1250704.

Minamino T, Kitakaze M (2010). ER stress in cardiovascular disease. Journal of Molecular and Cellular Cardiology 48: 1105-1110. DOI 10.1016/j.yjmcc.2009.10.026.

Mizukami T, Orihashi K, Herlambang B, Takahashi S, Hamaishi M et al. (2010). Sodium 4-phenylbutyrate protects against spinal cord ischemia by inhibition of endoplasmic reticulum stress. Journal of Vascular Surgery 52: 15801586. DOI 10.1016/j.jvs.2010.06.172.

Oakes SA, Scorrano L, Opferman JT, Bassik MC, Nishino M et al. (2005). Proapoptotic BAX and BAK regulate the type 1 inositol trisphosphate receptor and calcium leak from the endoplasmic reticulum. Proceedings of the National Academy of Sciences of the United States of America 102: 105-110. DOI 10.1073/pnas.0408352102.

Ohri SS, Hetman M, Whittemore SR (2013). Restoring endoplasmic reticulum homeostasis improves functional recovery after spinal cord injury. Neurobiology of Disease 58: 29-37. DOI 10.1016/j.nbd.2013.04.021.

Ohri SS, Maddie MA, Zhang Y, Shields CB, Hetman M et al. (2012). Deletion of the pro-apoptotic endoplasmic reticulum stress response effector CHOP does not result in improved locomotor function after severe contusive spinal cord injury. Journal of Neurotrauma 29: 579-588. DOI 10.1089/ neu.2011.1940.

Ohri SS, Maddie MA, Zhao Y, Qiu MS, Hetman M et al. (2011). Attenuating the endoplasmic reticulum stress response improves functional recovery after spinal cord injury. Glia 59: 1489-1502. DOI 10.1002/glia.21191.

Penas C, Guzman MS, Verdu E, Fores J, Navarro X et al. (2007). Spinal cord injury induces endoplasmic reticulum stress with different cell-type dependent response. Journal of Neurochemistry 102: 1242-1255. DOI 10.1111/j.1471-4159.2007.04671.x.

Penas C, Verdu E, Asensio-Pinilla E, Guzman-Lenis MS, HerrandoGrabulosa M et al. (2011). Valproate reduces CHOP levels and preserves oligodendrocytes and axons after spinal cord injury. Neuroscience 178: 33-44. DOI 10.1016/j. neuroscience.2011.01.012.

Ron D, Walter P (2007). Signal integration in the endoplasmic reticulum unfolded protein response. Nature Reviews Molecular Cell Biology 8: 519-529. DOI 10.1038/nrm2199.

Rubovitch V, Barak S, Rachmany L, Goldstein RB, Zilberstein Y et al. (2015). The neuroprotective effect of salubrinal in a mouse model of traumatic brain injury. Neuromolecular Medicine 17: 58-70. DOI 10.1007/s12017-015-8340-3.

Schroder M, Kaufman RJ (2005a). ER stress and the unfolded protein response. Mutation Research/Fundamental and Molecular Mechanisms of Mutagenesis 569: 29-63. DOI 10.1016/j. mrfmmm.2004.06.056.

Schroder M, Kaufman RJ (2005b). The mammalian unfolded protein response. Annual Review of Biochemistry 74: 739-789. DOI 10.1146/annurev.biochem.73.011303.074134.

Thuret S, Moon LD, Gage FH (2006). Therapeutic interventions after spinal cord injury. Nature Reviews Neuroscience 7: 628-643. DOI $10.1038 / \mathrm{nrn} 1955$.

Valenzuela V, Collyer E, Armentano D, Parsons GB, Court FA et al. (2012). Activation of the unfolded protein response enhances motor recovery after spinal cord injury. Cell Death \& Disease 3: e272. DOI 10.1038/cddis.2012.8.

Valenzuela V, Onate M, Hetz C, Court FA (2016). Injury to the nervous system: A look into the ER. Brain Research 1648: 617-625. DOI 10.1016/j.brainres.2016.04.053.

Zhang HY, Wang ZG, Lu XH, Kong XX, Wu FZ et al. (2015). Endoplasmic reticulum stress: relevance and therapeutics in central nervous system diseases. Molecular Neurobiology 51: 1343-1352. DOI 10.1007/s12035-014-8813-7.

Zhang HY, Zhang X, Wang ZG, Shi HX, Wu FZ et al. (2013). Exogenous basic fibroblast growth factor inhibits ER stressinduced apoptosis and improves recovery from spinal cord injury. CNS Neuroscience \& Therapeutics 19: 20-29. DOI $10.1111 / \mathrm{cns} .12013$.

Zhang Z, Chen J, Chen F, Yu D, Li R et al. (2017). Tauroursodeoxycholic acid alleviates secondary injury in the spinal cord via up-regulation of CIBZ gene. Cell Stress Chaperones 23: 1-10.

Zhou Y, Ye L, Zheng B, Zhu S, Shi H et al. (2016). Phenylbutyrate prevents disruption of blood-spinal cord barrier by inhibiting endoplasmic reticulum stress after spinal cord injury. American Journal of Translational Research 8: 1864-1875. 